\title{
A Novel Experiment Searching for the Lepton Flavor Violating Decay $\mu \rightarrow$ eee
}

\section{Wiedner*, H. Augustin, S. Bachmann, N. Berger, M. Kiehn, A.-K. Perrevoort and A. Schöning}

Physikalisches Institut der Universität Heidelberg, INF 226, 69120 Heidelberg, Germany

E-mail: dwiednerdcern.ch

\section{Perić}

Institut für Technische Informatik, B6, 26, 68131 Mannheim, Germany

\begin{abstract}
The Mu3e experiment is aimed at the measurement or exclusion of the charged lepton flavor violating decay $\mu^{+} \rightarrow e^{+} e^{-} e^{+}$with a branching ratio (BR) sensitivity of $10^{-16}$. The decay $\mu^{+} \rightarrow$ $e^{+} e^{-} e^{+}$is strongly suppressed in the standard model with a BR of $\ll 10^{-50}$, but enhanced to observable levels in many models for new physics. To reach this sensitivity the Mu3e detector has to have high rate capabilities of $>10^{9}$ events per second and excellent momentum, spacial and time resolution to suppress background. Two double layers of pixel sensors around a large stopping target in a $1 \mathrm{~T}$ field allow for precise vertex and momentum determination. As the decay electrons have a low momentum $(\leq 53 \mathrm{MeV} / \mathrm{c})$, an extremely light pixel detector design with $\mathrm{X} / \mathrm{X}_{0} \leq 0.1 \%$ per layer will be realized with the help of high voltage monolithic active pixel sensors (HV-MAPS) thinned to $50 \mu \mathrm{m}$ in order to minimize the effects of multiple scattering. The detector is complemented by two timing systems, which allow to suppress combinatorial background even at high muon stop rates $>1 \mathrm{GHz}$.
\end{abstract}

The 21st International Workshop on Vertex Detectors

16-21 September 2012

Jeju, Korea

\footnotetext{
* Speaker.
} 


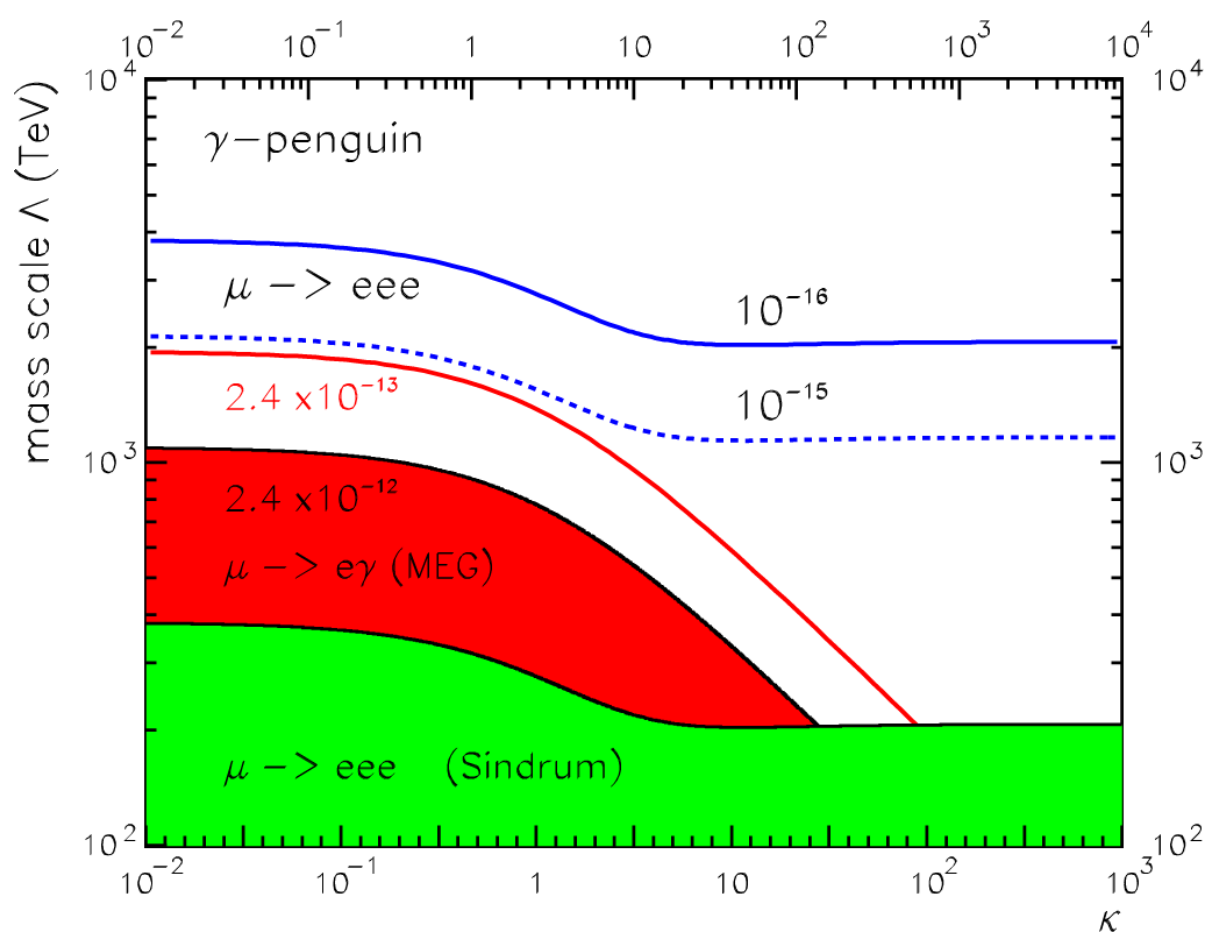

Figure 1: Experimental limits and projected limits on the LFV mass scale $\Lambda$ as a function of parameter $\kappa$ (see equation below), taken from [1], after a figure by A. de Gouvêa [13]

\section{Theory}

Charged lepton flavor violation is forbidden in the standard model (SM) of particle physics at tree level but can be introduced by lepton mixing in higher order loop diagrams. The dominant SM loop diagram is the one of neutrino mixing (figure 2) which is suppressed to a branching ratio of $\ll 10^{-50}$, which means in turn a high sensitivity to beyond standard model (BSM) lepton flavor violating reactions, see figure 3 and 4 . Examples for potential new physics models include inverse SEESAW SUSY models [2], super-symmetric $S U(3)_{c} \times S U(2)_{L} \times U(1)_{B-L} \times U(1)_{R}$ [3, 4], Little Higgs models with T-parity (LHT) [5, 6, 7, 8], Left-Right Symmetric models with additional Higgs triplets [9], models with new heavy Z-bosons or models with extra dimensions $[10,11,12]$. Introducing a common scale $\Lambda$ and a relative strength $\kappa$ between the dipole term and the 4-fermion contact interaction gives a simplified Lagrangian (1.1) [13, 14]. The mass reach of the indirect search via the rare decay $\mu^{+} \rightarrow e^{+} e^{-} e^{+}$can be expressed as a function of the relative strength $\kappa$, see figure 1 . The search for the decay $\mu^{+} \rightarrow e^{+} e^{-} e^{+}$is of fundamental interest as it is sensitive to new heavy particles beyond the reach of direct observation at the LHC and it is highly complementary to the searches for the decay $\mu \rightarrow \mathrm{e} \gamma$ and $\mu \rightarrow e$ conversion.

$$
\begin{aligned}
L_{L F V}= & \frac{m_{\mu}}{(\kappa+1) \Lambda^{2}} A_{R} \overline{\mu_{R}} \sigma^{\mu v} e_{L} F_{\mu v}+ \\
& \frac{\kappa}{(\kappa+1) \Lambda^{2}}\left(\overline{\mu_{L}} \gamma_{\mu} e_{L}\right)\left(\overline{e_{L}} \gamma^{\mu} e_{L}\right)
\end{aligned}
$$




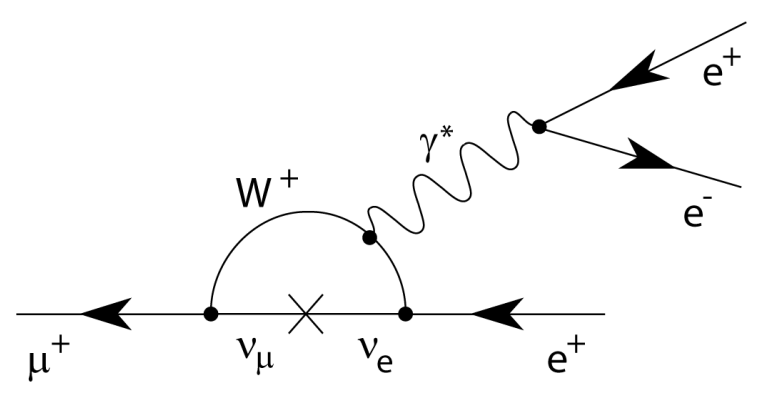

Figure 2: Feynman diagram for the $\mu^{+} \rightarrow e^{+} e^{-} e^{+}$process via neutrino mixing (indicated by the cross).

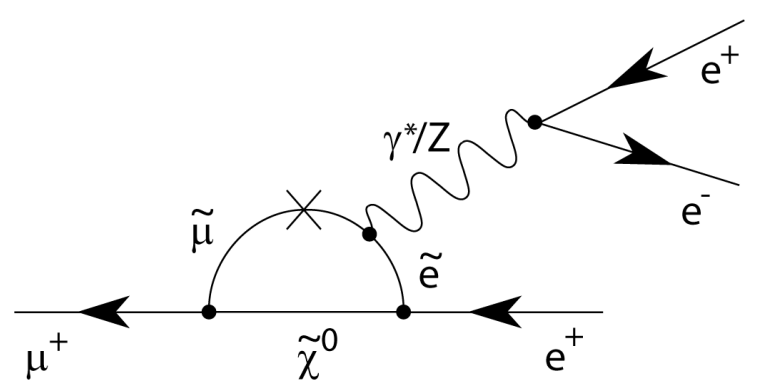

Figure 3: Diagram for lepton flavour violation involving supersymmetric particles.

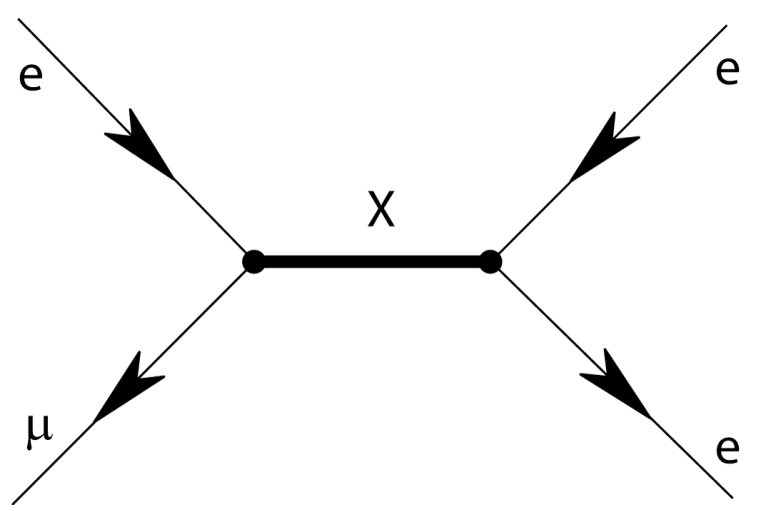

Figure 4: Diagram for lepton flavour violation at tree level.

\subsection{Background}

There are two main sources of background events, the radiative decay with internal conversion $\mu^{+} \rightarrow e^{+} e^{-} e^{+} \bar{v}_{\mu} v_{e}$ (BR 3.4 $10^{-5}$ ) [15] and combinatorial background. The combinatorial background stems from Michel decay positrons $\left(\mu^{+} \rightarrow e^{+} \bar{v}_{\mu} v_{e}\right)$ together with electrons from Bhabha scattering, internal conversion or misidentified positrons. The internal conversion background can only be identified through the missing energy carried away by the neutrinos which is achieved by precisely measuring the momentum of the decay products. The combinatorial background can be suppressed by precise vertex separation and exact decay time determination. 


\section{Challenges}

In order to reach the branching ratio sensitivity of $10^{-16}$ the Mu3e experiment faces multiple challenges. Examining more than $10^{16}$ muon decays in reasonable time of a few running years leads to high decay rates of $\mathscr{O}(\mathrm{GHz})$, so the detector must have high rate capabilities. To suppress the combinatorial background an excellent time resolution of $\leq 100 \mathrm{ps}$ and a good vertex separation of $200 \mu \mathrm{m}$ is required. The identification of background from the decay with internal conversion process $\mu^{+} \rightarrow e^{+} e^{-} e^{+} \bar{v}_{\mu} v_{e}$ can only be achieved with a very good momentum resolution of $\mathscr{O}(0.5) \mathrm{MeV} / \mathrm{c}$. Both the vertex and the momentum requirement lead to the need for an extremely low material budget in the active volume to minimize multiple scattering.

\section{Detector Concept}

The Mu3e experiment studies the decay of positive muons at rest, slowed down by energy loss on an aluminum target. The dominant standard model decays will be $\mu^{+} \rightarrow e^{+} \bar{v}_{\mu} v_{e}$ (Michel Decay), $\mu^{+} \rightarrow e^{+} \bar{v}_{\mu} v_{e} \gamma$ (radiative decay, BR $1.4 \cdot 10^{-2}$ for $E_{\gamma}>10 \mathrm{MeV}$ ) and with BR $3.4 \cdot 10^{-5}$ $\mu^{+} \rightarrow e^{+} e^{-} e^{+} \bar{v}_{\mu} v_{e}$ (internal conversion) [15]. Since the muons decay at rest the energy sum of the decay particles is given by the muon mass.

\subsection{Muon Beam}

The muon beam for the proposed Mu3e experiment must be of very high intensity. The most intense continuous muon beam with $>10^{8}$ muon decays per second is currently available at the Paul Scherrer Institute in Switzerland. This beam is produced as a surface muon beam in a thick carbon target using the high intensity $2.2 \mathrm{~mA}$ proton beam with $590 \mathrm{MeV} / \mathrm{c}$ and will be used in the phase I of the Mu3e experiment, see figure 5. To reach the ultimate sensitivity of $\mathrm{BR} \leq 10^{-16}$ a new beam-line will be required. The proposed High Intensity Muon Beam-line (HiMB) would make use of surface muons created in the target of the Swiss Spallation Neutron Source (SINQ) and could deliver muon decay rates in excess of $2 \cdot 10^{9} / \mathrm{s}$ for the phase II of the experiment. 


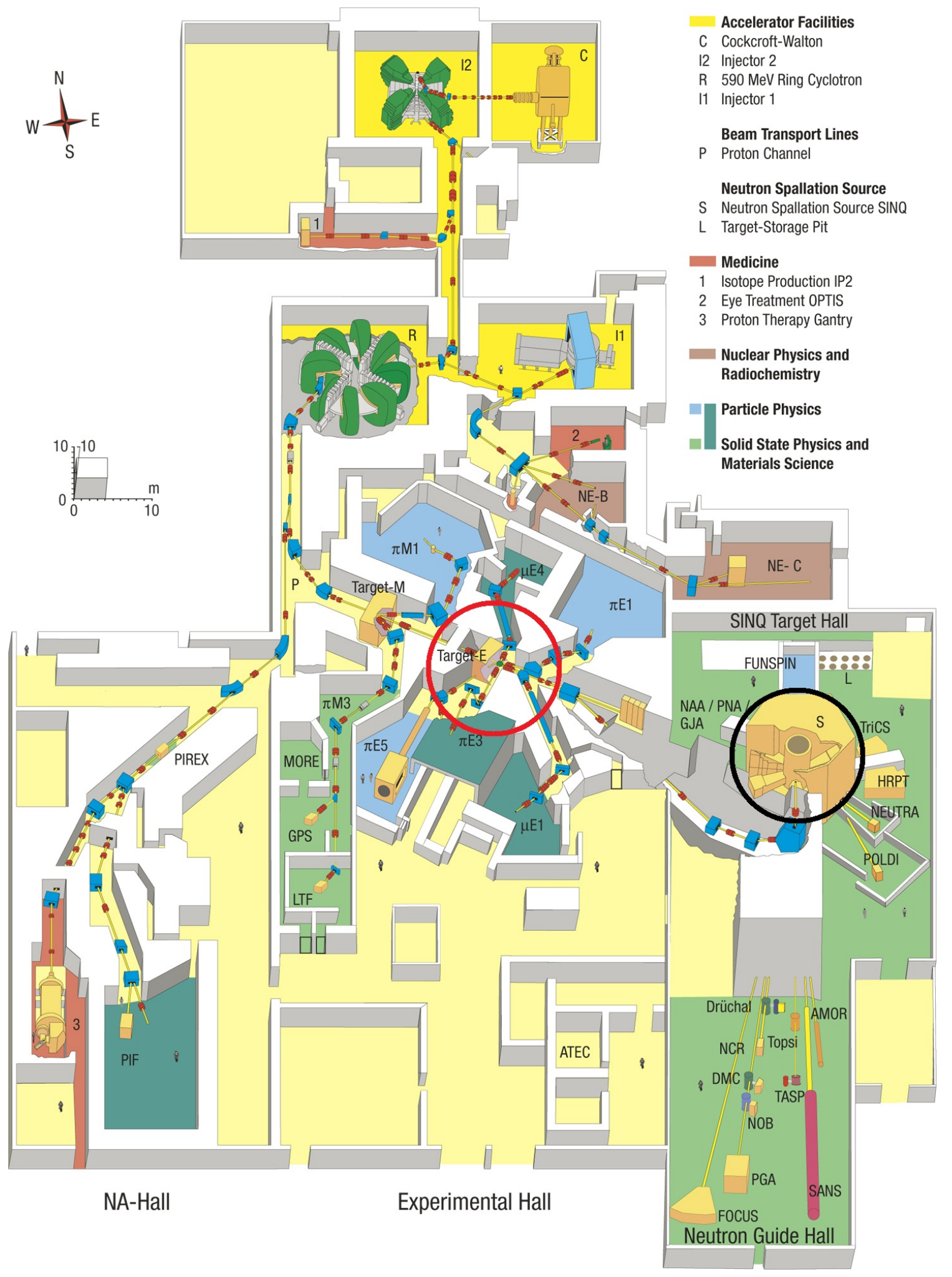

Figure 5: Overview of the experimental hall at the Paul Scherrer Institute. The existing beam-line starts at target E (red circle), whereas the high intensity beam-line would use muons produced in the spallation neutron source (black circle). 


\subsection{Long Tube Design}

It can be shown that the multiple scattering effects on momentum measurement cancel to first order if a particle track is measured before and after half a turn, see figure 6 . For this reason the Mu3e pixel detector consists of a $36 \mathrm{~cm}$ long central pixel detector around a double cone target which is extended to both sides by two silicon pixel stations to measure the recurling particles, see figure 7. The pixel detector allows for good vertex reconstruction and together with the surrounding solenoid magnet of $1 \mathrm{~T}$ strength, a very good momentum resolution of $\mathrm{RMS}(\mathrm{p})=0.28 \mathrm{MeV} / \mathrm{c}$ can be achieved which has been shown in simulation [1]. The tracking detector system is supplemented by two timing detector systems, the fiber detector and the tile detector.

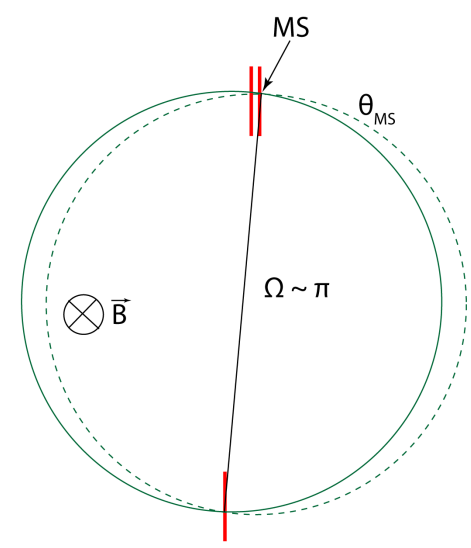

Figure 6: To first order multiple scattering (MS) effects on momentum measurements cancel, if the particle trajectory is measured before and after a $180^{\circ}$ turn.

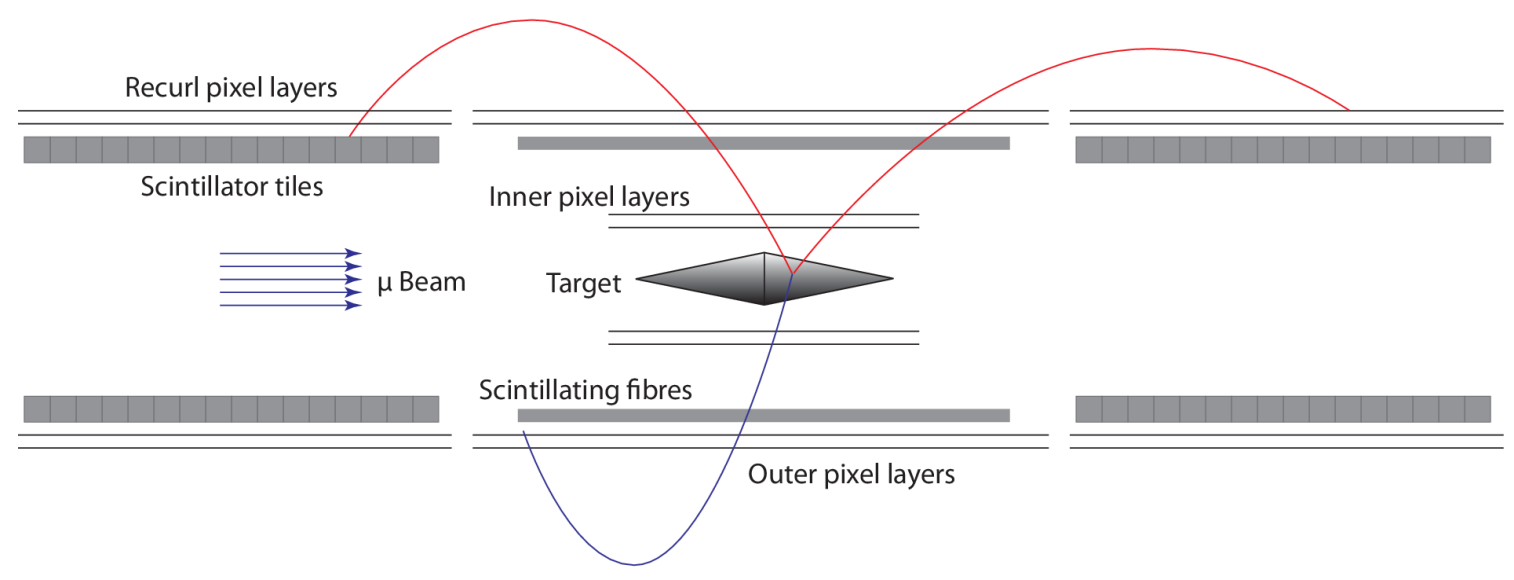

Figure 7: Schematic view of the Mu3e detector 


\subsection{Target}

The target has a large surface in order to make the separation of decay events possible. It is of double cone shape with $10 \mathrm{~cm}$ total length and $2 \mathrm{~cm}$ diameter. The target is made from aluminum foil with $30 \mu \mathrm{m}$ thickness for the front and $80 \mu \mathrm{m}$ for the rear cone, which results in equally spread decays and little multiple scattering for decay particles traversing the target.

\subsection{HV-MAPS}

High voltage monolithic active pixel sensors combine the advantages of classical hybrid pixel sensors with built-in analog and digital front-end electronics [16, 17]. Because the charge collection in the high electric field is drift based, a time resolution of $10 \mathrm{~ns}$ is possible, which is important for suppression of combinatorial background at high event rates. The implementation of both analog and digital electronics inside a deep $\mathrm{N}$-well makes extra readout chips in the active region unnecessary. This together with the possibility to thin the HV-MAPS to $50 \mu \mathrm{m}$ without loss of signal, leads to a pixel layer design with very low radiation length. The output data of the HVMAPS is fully zero-suppressed and serialized, so only $\mathscr{O}(30)$ wire-bonds are necessary per chip. There are two types of HV-MAPS, one with a size of $1.1 \mathrm{~cm} \times 2 \mathrm{~cm}$ for the vertex layers around the target, and the other with $2 \mathrm{~cm} \times 2 \mathrm{~cm}$ size for all outer and recurl layers. The pixel size for all layers is $80 \mu \mathrm{m} \times 80 \mu \mathrm{m}$.

So far two versions of HV-MAPS for the Mu3e experiment have been extensively tested [18, 19]. These prototypes with $42 \times 36$ pixels of $30 \mu \mathrm{m} \times 39 \mu \mathrm{m}$ size have shown a good signal to noise ratio $>20$ and a reasonable double pulse resolution of $2.7 \mu \mathrm{s}$. In the mean time the MUPIX3 chip has been produced, which is a larger prototype with $40 \times 32$ pixels of $80 \mu \mathrm{m} \times 92 \mu \mathrm{m}$ size and the first HV-MAPS using a zero-suppression scheme.

\subsection{Pixel Tracker}

The pixel detector is composed of inner double layers around the target, which together with an outer double layer form the central pixel station. Copies of the outer double layers form two recurl stations in front and two recurl stations after the central pixel station. The inner double layers have a length of $12 \mathrm{~cm}$ and a radius of $1.9 \mathrm{~cm}$ and $2.9 \mathrm{~cm}$. They are used for vertex reconstruction and as all other pixel layers for the momentum determination. The outer double layers have a length of $36 \mathrm{~cm}$ and a radius of $7.3 \mathrm{~cm}$ and $8.5 \mathrm{~cm}$. With the HV-MAPS thinned to $50 \mu \mathrm{m}$ a radiation length of $\mathrm{X} / \mathrm{X}_{0} \leq 0.1 \%$ can be achieved, leading to a good track resolution of $\mathscr{O}(200) \mu \mathrm{m}$. It is foreseen to run with the central tracking station and the first pair of recurl stations in phase I of the experiment and adding the second pair of recurl stations for the phase II. While the central pixel station alone provides reasonable momentum resolution of $\operatorname{RMS}(\mathrm{p})=0.73 \mathrm{MeV} / \mathrm{c}$, the measurement of recurling tracks with all four recurl stations gives a momentum resolution of $\mathrm{RMS}(\mathrm{p})=0.28 \mathrm{MeV} / \mathrm{c}$. The full pixel tracker is $\approx 2 \mathrm{~m}$ long and has $\mathscr{O}(280)$ million pixel cells.

\subsection{Timing Detectors}

The two timing detectors are necessary to suppress combinatorial background at the high muon decay rates. The fiber detector is a cylindrical time of flight system of $36 \mathrm{~cm}$ length and at a radius of $6 \mathrm{~cm}$ around the target. It can provide timing information even for low energetic decay products 
with an accuracy of $\mathscr{O}(1)$ ns. Since it sits in the active volume it is optimized for low radiation length to minimize multiple scattering. Three to five layers of $250 \mu \mathrm{m}$ thin scintillating fibers are under investigation.

The tile detector is the second time of flight system. It is mounted inside the four pixel recurl stations at a radius of $6 \mathrm{~cm}$. The tile detector consists of scintillating plastic tiles of around $7.5 \times 7.5 \times 5 \mathrm{~mm}^{3}$ size. As the charged decay particles hit the relatively thick tiles a lot of photons are produced, leading to a very good time resolution of $<100 \mathrm{ps}$. Both timing detectors employ SiPMs which operate in the $1 \mathrm{~T}$ magnetic field and have a good photon detection efficiency and time resolution.

\subsection{Mechanics}

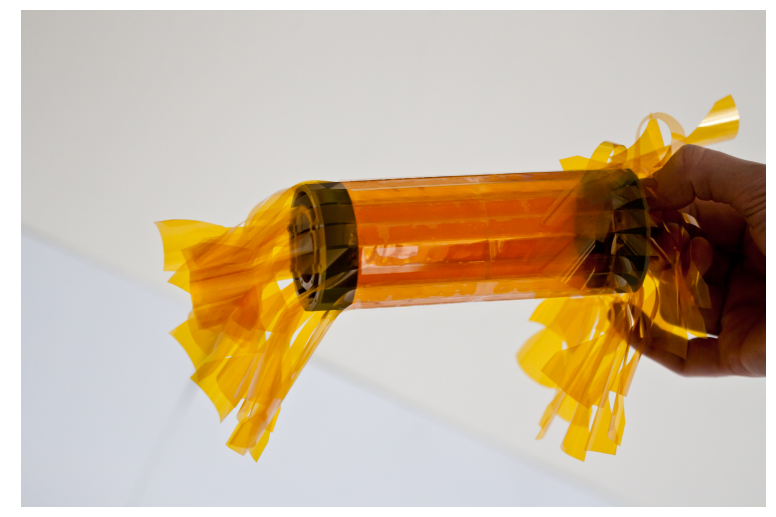

Figure 8: Mechanical support structure for the vertex tracker

The mechanical support of the pixel tracker in the active volume has been optimized for extremely low radiation length, minimizing multiple scattering. The HV-MAPS are thinned to $50 \mu \mathrm{m}$ and then glued and wire bonded to a $25 \mu \mathrm{m}$ thick flex-print with aluminum traces. The flex-print with the chips is then mounted on a mechanical frame made from $25 \mu$ m thick Kapton ${ }^{\circledR}$ foil. Figure 8 shows a mechanical prototype build from $25 \mu \mathrm{m}$ thick Kapton ${ }^{\circledR}$ foil and $100 \mu \mathrm{m}$ thin glass plates, which is extremely light and surprisingly sturdy

\subsection{Readout}

The Mu3e experiment has a trigger-less continuous readout and a track based online event filter. The HV-MAPS chips use a $20 \mathrm{MHz}$ system clock to generate event timestamps for the hits. Hit data consisting of time-stamp and pixel address is serialized and send over multiple $800 \mathrm{Mbit} / \mathrm{s}$ serial LVDS links to front-end FPGAs. Each front-end FPGA then sends long frame trains over optical links to the read-out boards. In the read-out boards data from many front-end FPGAs is collected and send over $10 \mathrm{Gbit} / \mathrm{s}$ optical links to the 50 event filter farm PCs. There data is received and assembled by another FPGA board and send via PCIe to a graphical processing unit (GPU). The GPUs perform a track based event selection. The amount of data stored will be in the order of $50 \mathrm{MBytes} / \mathrm{s}$. 


\section{Schedule}

The Mu3e research proposal has been submitted to the Paul Scherrer Institute in late 2012 [1] and the experiment was accepted in early 2013. In 2013 and 2014 the R\&D for the pixel detector will be carried out and the central pixel station will be assembled. In 2015 the central detector will be commissioned and the run of phase IA at a beam intensity of a few $10^{7}$ muons per second will start. In the same year the first recurl stations, the fiber tracker and the first pair of tile detectors will be assembled and tested. In 2016 the phase I detector will be completed and a physics run with $10^{8}$ muons per second will be taken. By 2017 the remaining recurl and tile detector stations will be ready and the Mu3e detector can be completed. Depending on the availability of the high intensity beam-line the phase II physics run will start in 2017 or 2018.

\section{Summary}

The proposed Mu3e experiment will search for the lepton flavor violating decay $\mu^{+} \rightarrow e^{+} e^{-} e^{+}$ with an ultimate branching ratio sensitivity of $10^{-16}$. Any measurement of the decay $\mu^{+} \rightarrow e^{+} e^{-} e^{+}$ would be a sign for new physics. A rate of $10^{9}$ muons per second and a very good background rejection are required to reach the projected sensitivity. The Mu3e detector is based on a silicon pixel tracker around a large target in a $1 \mathrm{~T}$ solenoidal field, complemented by two types of timing detectors. By using novel high voltage active pixel sensors the radiation length of a tracking layer can be kept extremely low with $\mathrm{X} / \mathrm{X}_{0} \leq 0.1 \%$, leading to a momentum resolution of $\mathrm{RMS}(\mathrm{p})=0.28 \mathrm{MeV} / \mathrm{c}$. A research proposal has been submitted to the Paul Scherrer Institute end of 2012 and physics data taking could start in 2015 .

\section{References}

[1] A. Blondel et al., Research Proposal for an Experiment to Search for the Decay $\mu \rightarrow$ eee, December 2012, Available at: www.psi.ch/mu3e/DocumentsEN/ResearchProposal .pdf

[2] A. Abada, D. Das, A. Vicente and C. Weiland, Enhancing lepton flavour violation in the supersymmetric inverse seesaw beyond the dipole contribution, JHEP 1209 (2012) 015 [hep-ph/1206.6497].

[3] M. Malinsky, J.C. Romao and J.W.F Valle, Novel supersymmetric SO(10) seesaw mechanism, Phys.Rev.Lett. 95 (2005) 161801 [hep-ph/ 0506296 ].

[4] V. De Romeri, M. Hirsch, M. Malinsky, Soft masses in SUSY SO(10) GUTs with low intermediate scales, Phys.Rev. D84 (2011) 053012 [hep-ph/1107. 3412].

[5] F. del Aguila, J.I. Illana and M.D. Jenkins, Precise limits from lepton flavour violating processes on the Littlest Higgs model with T-parity, JHEP 0901 (2009) 080 [hep-ph/ 0811 . 2891].

[6] F. del Aguila, J.I. Illana and M.D. Jenkins, Lepton flavor violation in the Simplest Little Higgs model, JHEP 1103 (2011) 080 [hep-ph/1101.2936].

[7] M. Blanke, A.J. Buras, B. Duling, A. Poschenrieder and C. Tarantino, Charged Lepton Flavour Violation and $(\mathrm{g}-2)(\mathrm{mu})$ in the Littlest Higgs Model with T-Parity: A Clear Distinction from Supersymmetry, JHEP 0705 (2007) 013 [hep-ph / 0702136$].$ 
[8] M. Blanke, A.J. Buras, B. Duling, S. Recksiegel and C. Tarantino, FCNC Processes in the Littlest Higgs Model with T-Parity: a 2009 Look, Acta Phys. Polon. B41 (2010) 657-683 [hep-ph/0906.5454].

[9] M. Kakizaki, Y. Ogura and F. Shima, Lepton flavor violation in the triplet Higgs model, Phys.Lett. B566 (2003) 210-216 [hep-ph/ 0304254 ].

[10] L. Randall and R. Sundrum, A large mass hierarchy from a small extra dimension, Phys. Rev. Lett. 83 (1999) 3370 .

[11] N. Arkani-Hamed and M. Schmaltz, Hierarchies without symmetries from extra dimensions, Phys. Rev. D61 (2000) 33005.

[12] W.F. Chang and J.N. Ng, Lepton flavor violation in extra dimension models, Phys.Rev. D71 (2005) 053003 [hep-ph/0501161].

[13] A. de Gouvêa, (Charged) lepton flavor violation, Nucl. Phys. Proc. Suppl. 188 (2009) 303.

[14] Y. Kuno, Y. Okada, Muon decay and physics beyond the standard model, Rev. Mod. Phys. 73 (2001) 151-202 [hep-ph/9909265].

[15] K. Nakamura et al. [Particle Data Group], Review of particle physics, J. Phys. G37 (2010) 075021.

[16] I. Perić, A novel monolithic pixelated particle detector implemented in high-voltage CMOS technology, Nucl. Instrum. Meth. A 582 (2007) 876.

[17] I. Perić et al., Strip Technology and HVMAPS, this proceedings.

[18] H. Augustin, Charakterisierung von HV-MAPS, Bachelor thesis, University of Heidelberg 2012.

[19] A.-K. Perrevoort, Characterisation of High-Voltage MonolithicActive Pixel Sensors for the Mu3e Experiment, Master thesis, University of Heidelberg 2012. 